\title{
Mathematical Model of Airflow in the Lungs of Children II: Effects of Ventilatory Parameters
}

\author{
X. GUAN ${ }^{a}$, R.A. SEGAL ${ }^{\text {b. }}$, M. SHEARER ${ }^{b}$ and T.B. MARTONEN ${ }^{c,+}$ \\ ${ }^{a}$ Department of Medicine, Duke University, Durham, NC $27710,{ }^{b}$ Department of Mathematics, North Carolina State University, Raleigh, NC \\ 27695 and ${ }^{\mathrm{c}}$ National Health and Environmental Effects Research Laboratory, U.S. EPA, Mail Drop 74, RTP, NC 277II, and Division of Pul- \\ monary Diseases, Department of Medicine, University of North Carolina, Chapel Hill, NC 27599
}

(In final form September 9, 1999)

\begin{abstract}
In an effort to develop more effective aerosol therapy procedures, we examined airflow patterns in the lung of a child (age four years). In particular, we were concerned with how ventilatory parameters (i.e., breathing rate and tidal volume) affected the patterns of airflow around tumors. To conduct the study, a computational fluid dynamics package. FIDAP, was used to define a model lung. The results of simulations show the extent to which changing ventilatory parameters can affect flow patterns in the neighborhood of the tumors as well as drug distribution throughout the lung.
\end{abstract}

Keywords: Pediatric medicine, Mathematical model, Fluid dynamics, Aerosol therapy, Tumors, Ventilation Parameters

\section{INTRODUCTION}

To develop more effective aerosol therapy protocols for a variety of lung diseases (e.g., asthma, cystic fibrosis, and lung cancer), our laboratories have systematically addressed airflow patterns in human lungs. The underlying hypothesis of our work being that once fluid dynamics conditions within airway systems are understood, it will be possible to determine the trajectories and deposition patterns of pharmacological drug particles entrained in the air streams.

We have focused on drug delivery to tumors as a representative airway disease. There has been some research regarding the treatment of tumors with aerosol therapy by Morisson et al. (1993) and Tatsumura (1993). By conducting computer simulations of flow around tumor sites, we show that there is potential for targeted delivery of drugs to tumor locations. In Part I of this study, we documented the effects of sizes and locations of tumors on the motion of air within the lung; herein, we address ventilatory parameters.

To target the delivery of inhaled drugs, a technique at the physician's disposal is control of ventilation. This includes varying tidal volumes and breathing frequencies during spontaneous breathing trials and mechanical ventilation (Dhand and Tobin, 1997). Therefore, in this work we have examined the impact

* Supported by a CRAY grant through the North Carolina Supercomputing Center and by the ARO.

+ Corresponding author. Tel: (919)541-7875; Fax: (919)541-4284; E-mail: martonen.ted@epamail.epa.gov 


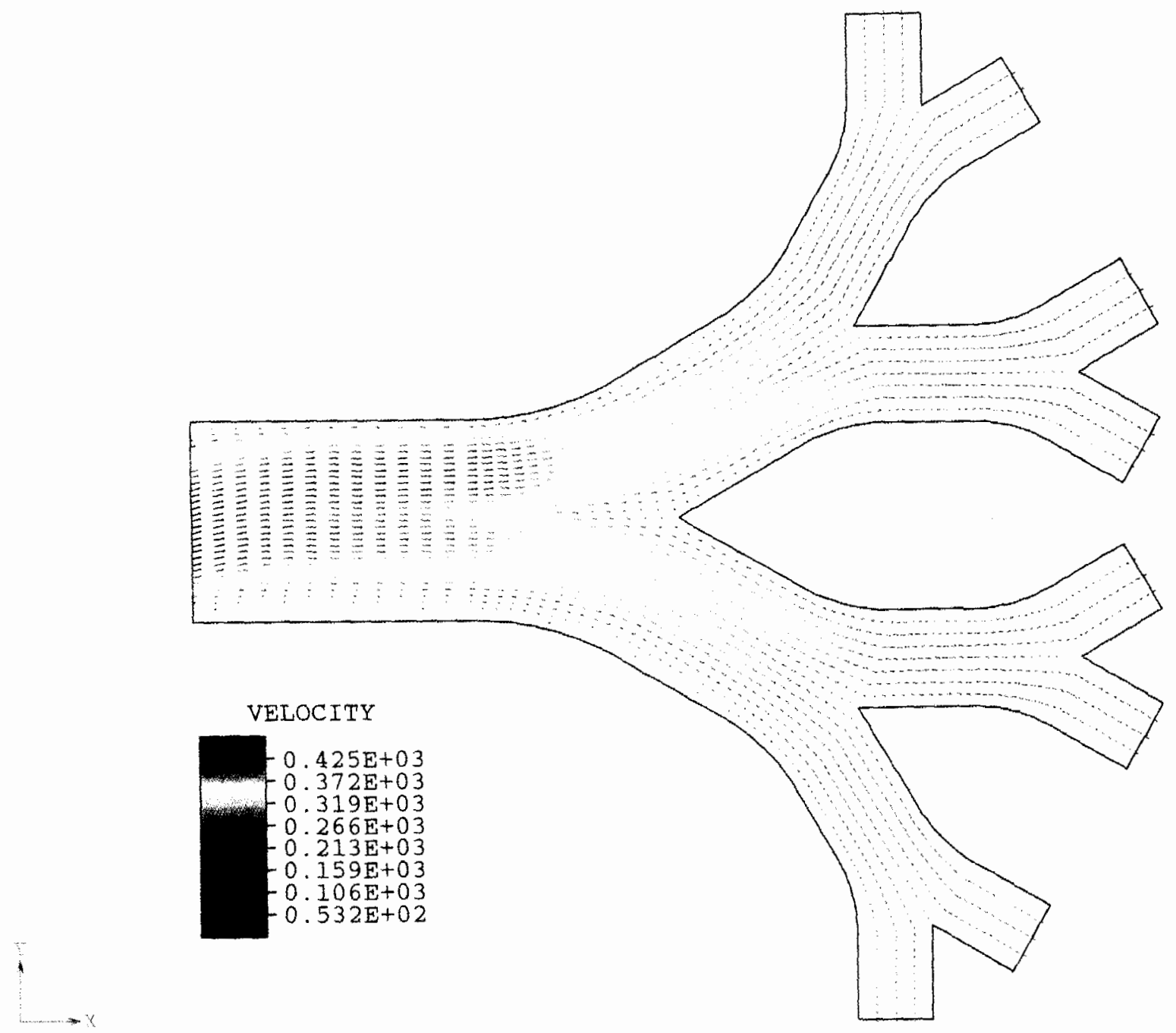

FIGURE 1 Velocity fields in generations $2 \leq I \leq 5$ of a four-year-old's lung with a skewed inlet velocity profile corresponding to a respiratory intensity level 3 breathing rate (see Table I) (See Color Plate I at the back of this issue)

of regulating ventilation on localized and bulk flow effects.

The simulations were performed using a computational fluid dynamics package, FIDAP (1993). We found that breathing patterns do indeed have pronounced effects on flow conditions around tumors, suggesting that physicians may elect to regulate ventilatory parameters to selectively deposit inhaled pharmacologic drugs.

\section{METHODS}

\section{Lung Morphology}

We have focused on the upper tracheobronchial airways of a child. The model was conceptually based on the symmetric lung morphology of Weibel (1963) for the adult, but was scaled to a four-year-old child's dimensions using the formulas of Hofmann et al. 


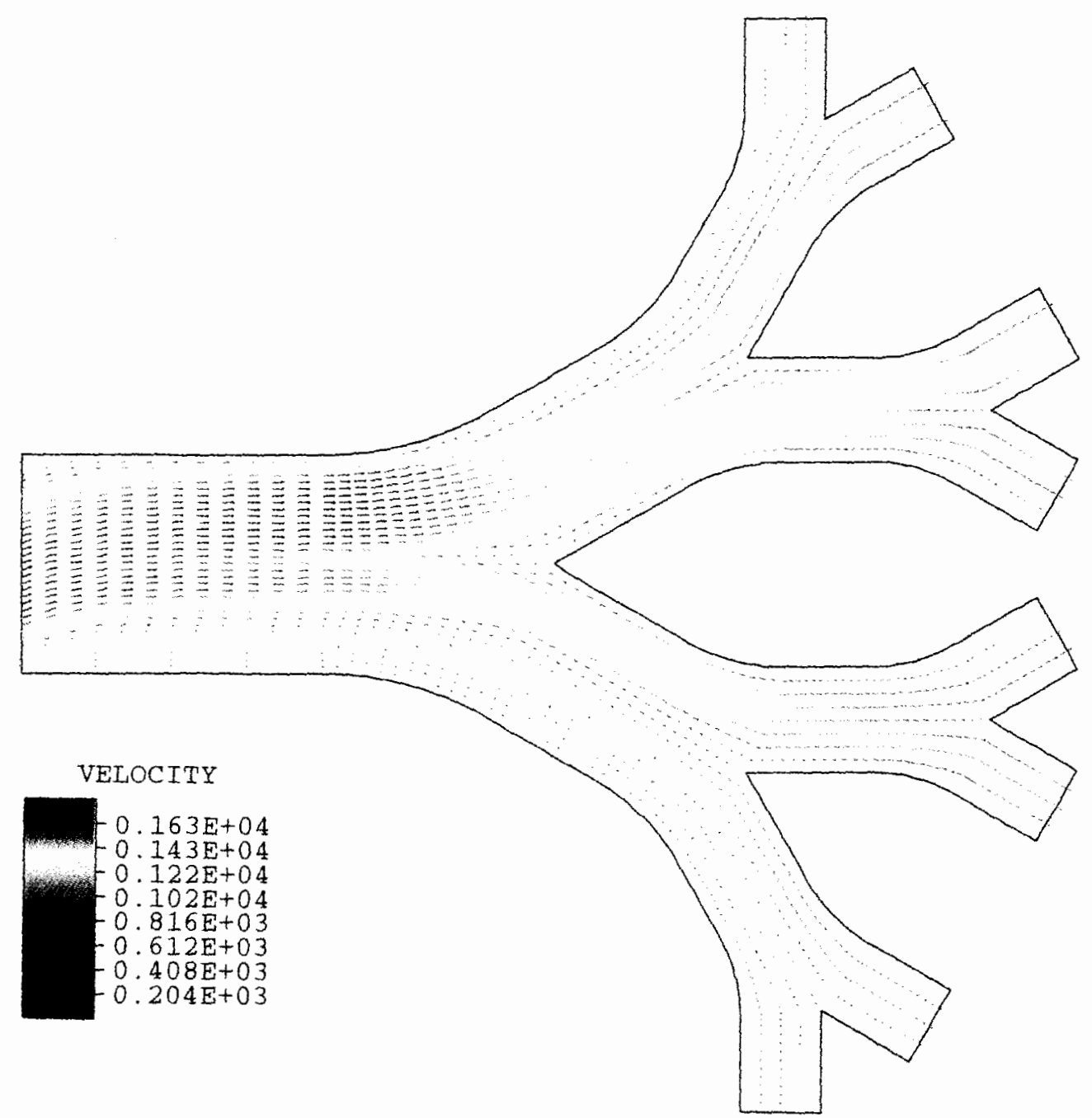

FIGURE 2 Velocity fields in generations $2 \leq I \leq 5$ of a four-year-old"s lung with a skewed inlet velocity profile corresponding to a respiratory intensity level 5 breathing rate (see Table I) (See Color Plate II at the back of this issue)

(1989). Based on the research of Cleveland (1979), all of the branching angles were set to $30^{\circ}$. Additional details regarding the morphology have been discussed in Part I.

The different tumor sizes and sites considered in our investigation have been presented in Figure 2 and Table II of Part I. These tumor sites were selected based on the works of Catley et al., 1951; Clements and Gavelle, 1986; Cohen et al., Condon, 1962; dePareder et al., 1970; Hartman and Shochat, 1983; Kirchner, 1951; Kramer et al., 1985; Nunez et al., 1966; Oho and Amemiya, 1980; Oleszczuk-Raszke and Cremin, 1988; Vawter and Ferguson, 1958; Wang et al., 1993; Weisel and Leplye, 1961; and Wellons et al., 1976. A representative selection of tumor distributions are analyzed below. 
TABLE I Ventilatory parameters for a four-year-old child

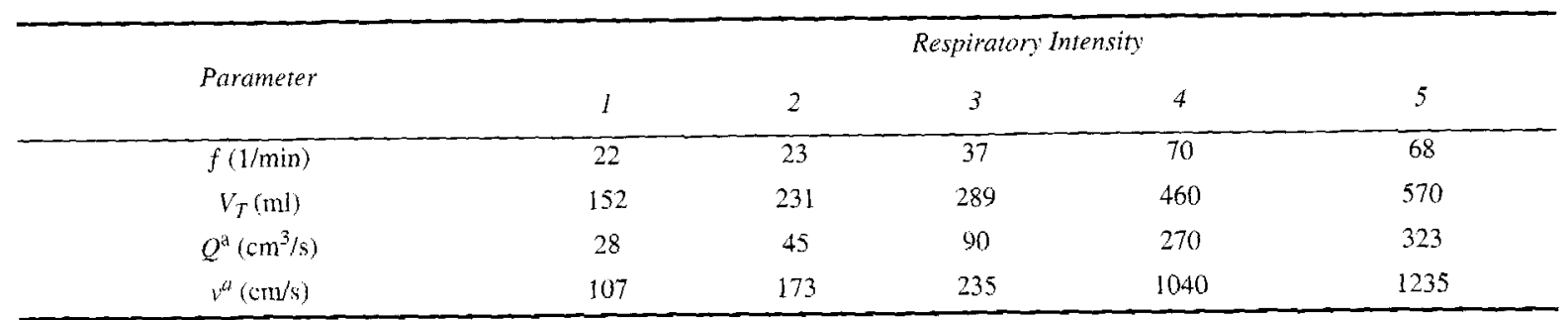

a. values are for gencration $I=2$

TABLE II Relationship of figures described in the Results section

\begin{tabular}{|c|c|c|c|c|}
\hline Part & Figure & Tumor Site & Respiratory Intensity & Control Case \\
\hline I & 9 & No tumor & L1 & - \\
\hline$I$ & 10 & Carinal ridge & $\mathrm{Ll}$ & Fig 9 \\
\hline 1 & 11 & Side wall & L1 & Fig 9 \\
\hline $\mathrm{J}$ & 13 & Carinal ridge, Side wall & $\mathrm{L} 1$ & Fig 9 \\
\hline II & 1 & Notumor & L3 & - \\
\hline II & 2 & No tumor & L5 & - \\
\hline II & 3 & Carinal ridge & $\mathrm{L3}$ & Fig 1 \\
\hline II & 4 & Carinal ridge & L5 & Fig 2 \\
\hline U] & 5 & Side wall & L3 & Fig 1 \\
\hline II & 6 & Side wall & L5 & Fig 2 \\
\hline II & 7 & Carinal ridge, Side wall & L3 & Fig 1 \\
\hline II & 8 & Carinal ridge, Side wall & LS & Fig 2 \\
\hline
\end{tabular}

\section{Velocity Profiles}

Five different levels of respiratory intensity were addressed; see Table I (Martonen et al., 1989). Values of breathing frequency $(f)$ and tidal volume $\left(\mathrm{V}_{T}\right)$ are presented for generation $\mathrm{l}=0$. These parameters defined the flow conditions for the simulations. As a matter of academic interest, the designated respiratory intensities 1,2,3,4 and 5 correspond to sedentary, low, light, heavy and maximal activity levels, respectively, and illustrate the wide range of human lung performance.

Also listed in Table $I$ are the corresponding inspiratory flow rates $(Q)$ and the average inlet velocities $(v)$. $Q$ was defined by the expression $Q=\left(V_{T} \cdot f \cdot 2\right) /(60$. 4). The factor 2 in the numerator denotes that only the inhalation portion of a breathing cycle is being described. The factor 4 is present in the denominator because there are $2^{l}=4$ airways in generation $\mathrm{I}=2$.
The velocity $(v)$ was determined by $v=Q /(\pi$. $\left.(0.575 / 2)^{2}\right)$, where $\pi \cdot(0.575 / 2)^{2}$ is the cross-sectional area of an airway in generation $\mathrm{I}=2$.

To be physiologically realistic, a skewed inlet velocity profile is used in this work as suggested by the results of Part I. This skewed profile was determined by beginning a simulation at generation $I=0$ with the appropriate flow (for brevity only Levels 1,3 or 5 were selected) and determining the corresponding velocity profile entering generation $I=2$ (see Part I, Figure 3).

\section{Fluid Dynamics}

The computations were performed using FIDAP (1993). This computational fluid dynamics package yields a numerical solution to the Navier-Stokes equations and has various graphics formats to facilitate 


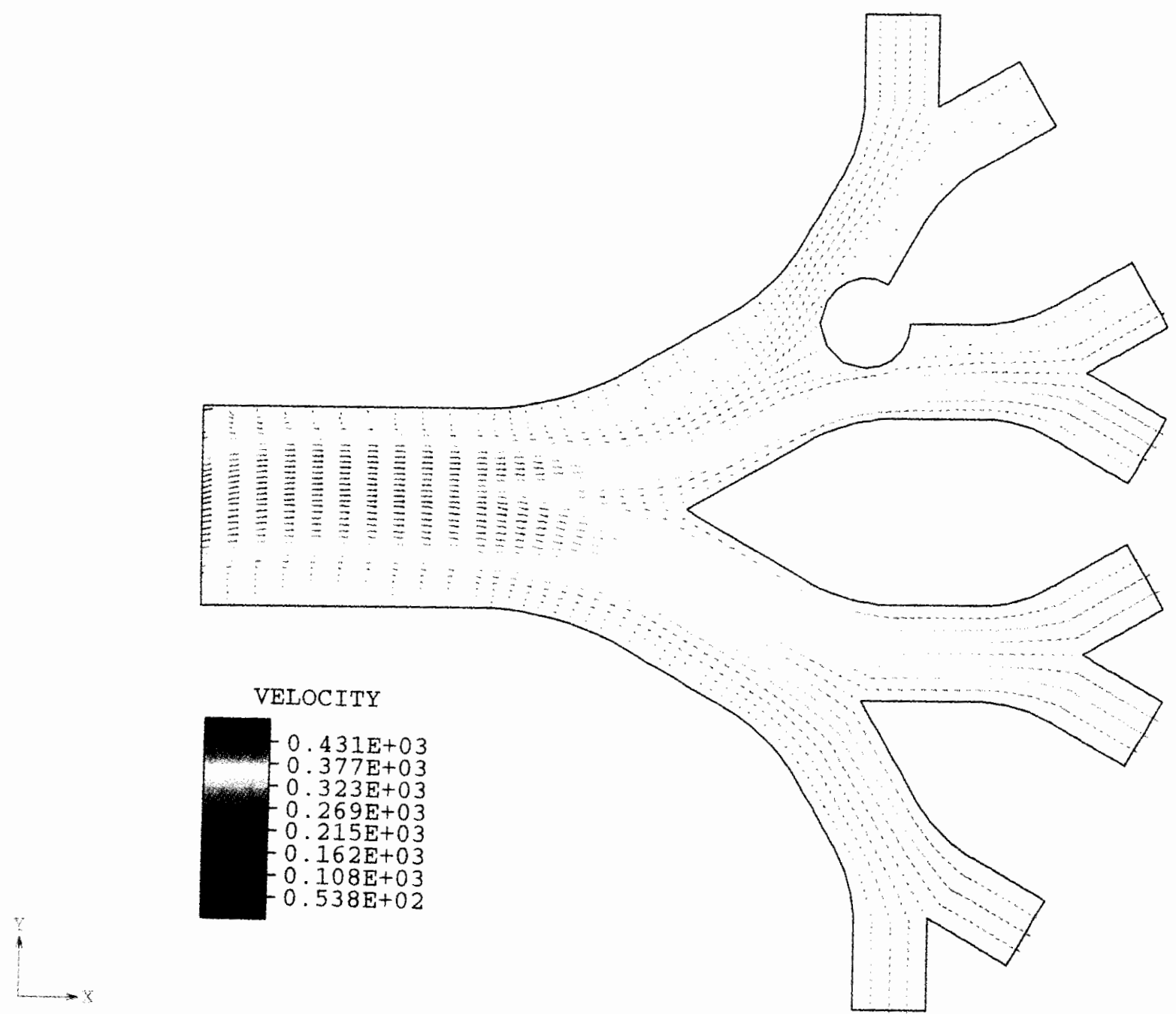

FIGURE 3 Velocity field in generations $2 \leq \mathrm{I} \leq 5$ of a four-year-old's lung with a skewed inlet velocity profile corresponding to a respiratory intensity level 3 breathing rate (see Table I). The tumor located on the carinal ridge has radius $0.131 \mathrm{~cm}$ (See Color Plate III at the back of
this issue)

visualizations of results using velocity fields, pressure contours and flow streamlines, etc. For additional details see Part I.

\section{RESULTS}

In the remainder of the paper, it will be useful to have a short-hand notation for identifying the different theoretical simulations. The simulations performed using initial velocity profiles corresponding to the breathing parameters for respiratory intensity levels 1, 3 and 5 of Table I will be denoted by L1, L3 and L5. Figures for the L3 and L5 simulations are presented below; the LI figures were presented in Part I. Table II is presented to orient the reader and organize the figures referred to in the text. The contents of Table II have two elements in common: $r / D=0.30$ (or, $r=0.131 \mathrm{~cm}$ ) and the inlet velocity profile is skewed. The airways will be referred to using the following convention: $\mathrm{I}^{n}$, 


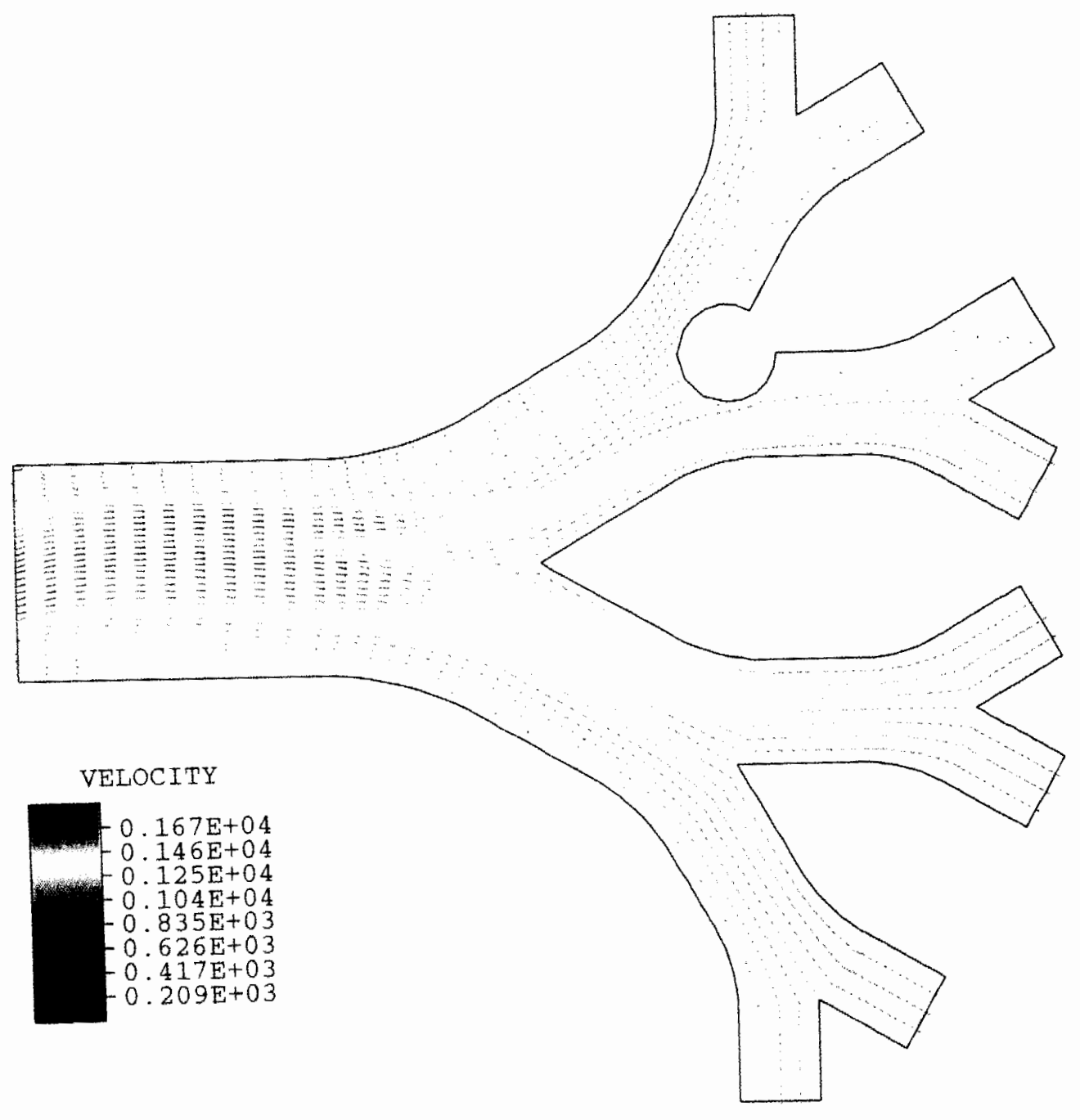

FIGURE 4 Velocity field in generations $2 \leq I \leq 5$ of a four-year-old's lung with a skewed inlet velocity profile corresponding to a respiratory intensity level 5 breathing rate (see Table I). The tumor located on the carinal ridge has radius $0.131 \mathrm{~cm}$ (See Color Plate IV at the back of this issue)

where I indicates the generation number and $n$ distinguishes the airways within each generation. For more details, see Figure 6 in Part I.

\section{No Tumors}

These simulations will serve as control cases with respect to which we can evaluate the results of other computer simulations. The effects of an increase in respiratory intensity were clear. The maximum velocities for L1, L3 and L5 conditions were $167 \mathrm{~cm} / \mathrm{s}$, $498 \mathrm{~cm} / \mathrm{s}$ and $1837 \mathrm{~cm} / \mathrm{s}$, respectively. Moreover, the increase in maximum velocity produced a maldistribution of flow within the airway network for the L5 simulation. This can be seen in Figure 2. The region $4^{a}$ had velocities which were approximately $75 \%$ slower than the velocities in region $4^{b}$. We did not observe this amount of variation between regions $4^{a}$ 


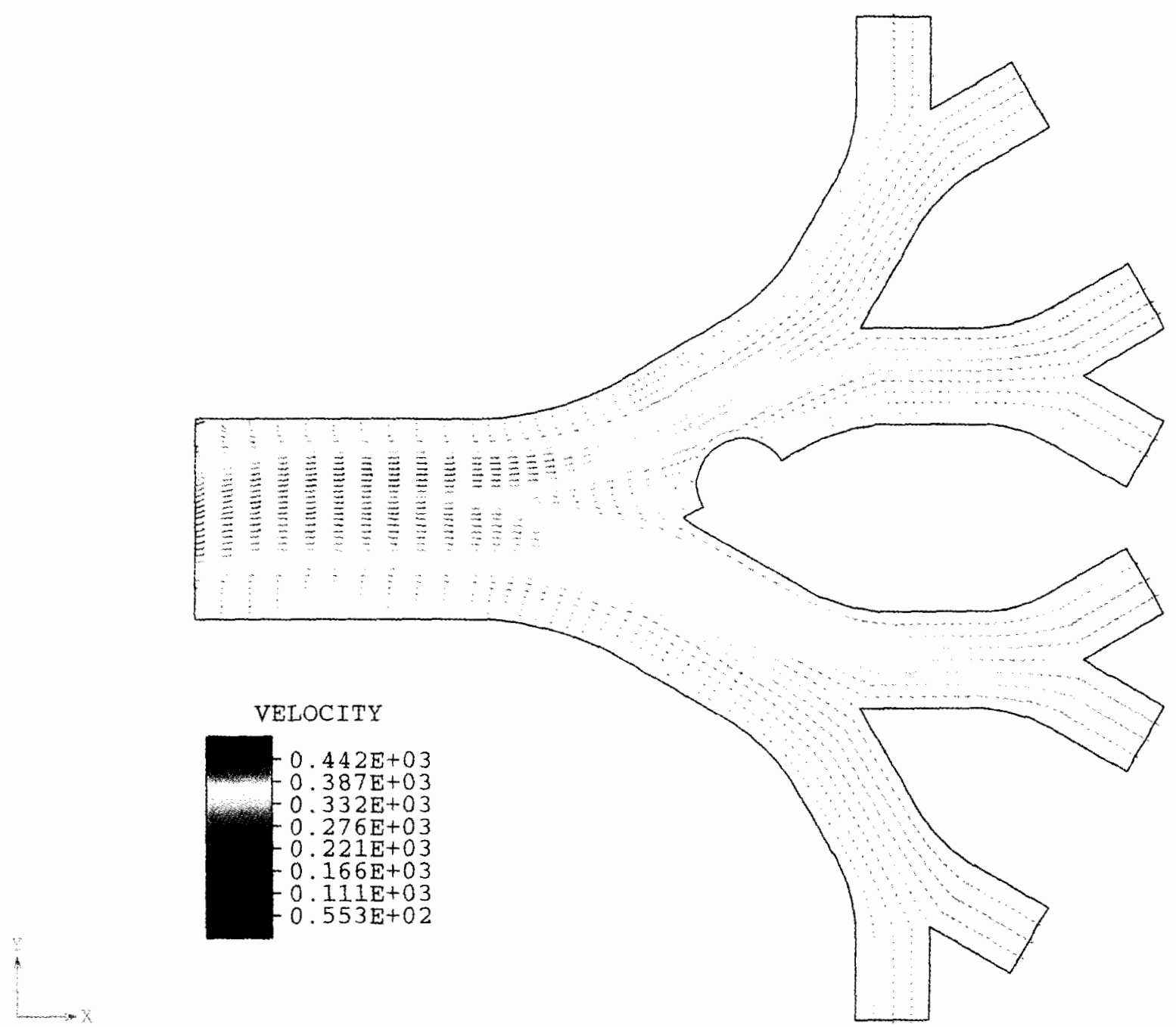

FIGURE 5 Velocity field in generations $2 \leq I \leq 5$ of a four-year-old's lung with a skewed inlet velocity profile corresponding to a respiratory intensity level 3 breathing rate (see Table I). The tumor located on the inside wall has a radius of $0.131 \mathrm{~cm}$ and is located $0.067 \mathrm{~cm}$ from the bifurcation point (See Color Plate $\mathrm{V}$ at the back of this issue)

and $4^{b}$ in the simulations for lower respiratory intensities (L1 and L3).

\section{Effects of an Isolated Tumor}

When a tumor was located in region $3^{b}$ (Figures 3-6, Part II; Figures 10-11, Part I) there was a change in flow volume distribution when compared to the respective control cases (Figures 1-2, Part II;
Figure 9 Part I). In all three cases, (L1, L3 and L5) the core flow (the red/orange/yellow region emanating from the inlet) was more pronounced in $3^{a}$ when a tumor was present than in the respective control cases.

\section{Tumor on the carinal ridge}

A tumor on a carinal ridge had the dominant effect on the overall motion of air in the network of airways. 


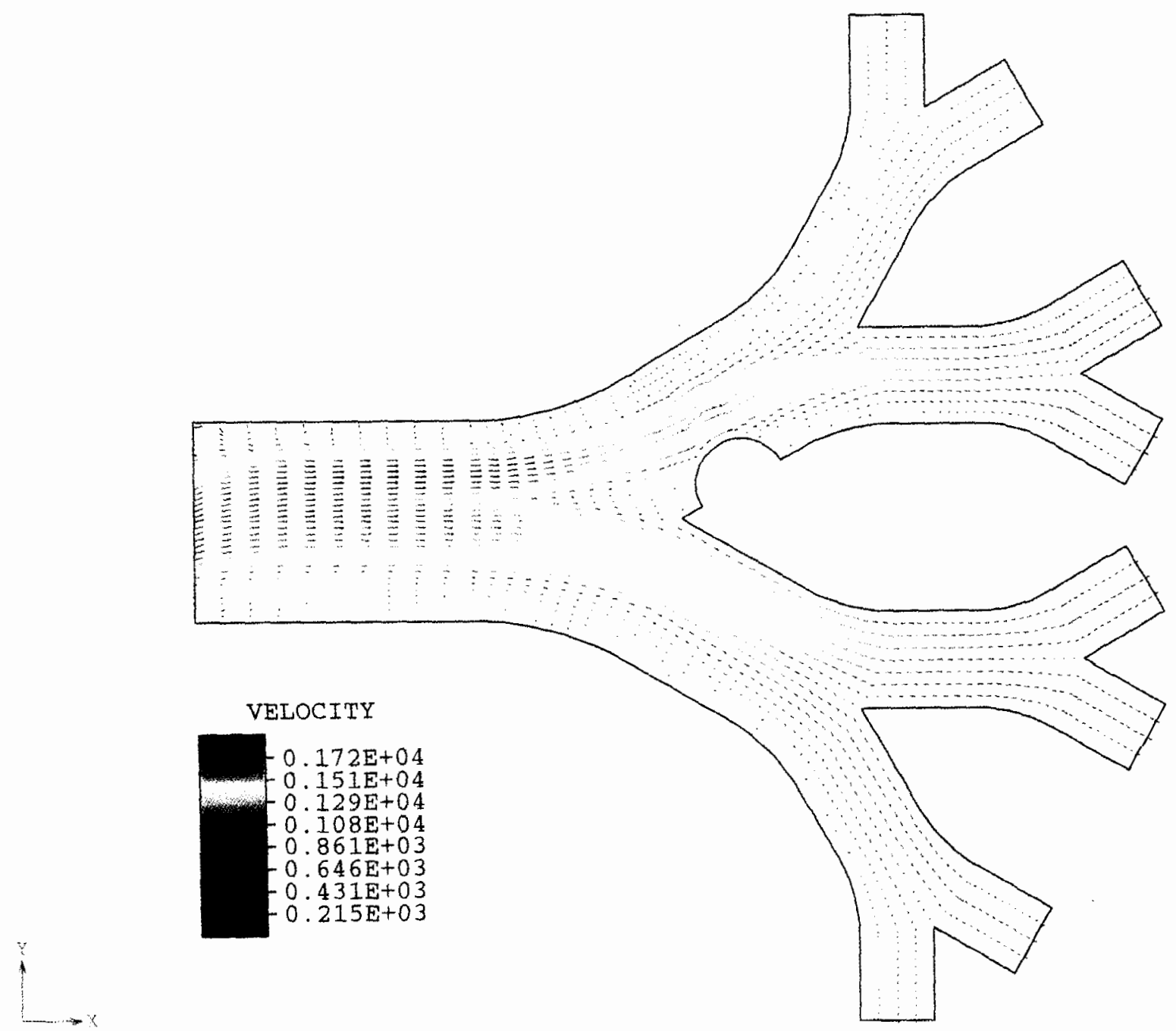

FIGLRE 6 Velocity field in generations $2 \leq I \leq 5$ of a four-year-old's lung with a skewed inlet velocity profile corresponding to a respiratory intensity level 5 breathing rate (see Table I). The tumor located on the inside wall has a radius of $0.131 \mathrm{~cm}$ and is located $0.067 \mathrm{~cm}$ from the bifurcation point (See Color Plate VI at the back of this issue)

Flow from the core of region 2 was primarily directed to region $3^{a}$ (see Figures 3 and 4 ). For the control cases (Figures 1 and 2 ) the core flow region was larger in region $3^{b}$. As the inlet flow rate was increased, this effect was enhanced (see Figure 10 in Part I and Figures 3 and 4 in Part II).

\section{Tumor on the inside wall}

For a given respiratory intenstiy, the presence of a side wall tumor affected the overall distribution of flow by reducing the effects of the skewed inlet velocity profile. That is, the core region was more evenly divided between region $3^{a}$ and region $3^{b}$ when compared to the corresponding control cases (compare Figure 1 [no tumor] with Figure 5 [tumor] for L3 breathing conditions and Figure 2 [no tumor] with Figure 6 [tumor] for L5 breathing conditions). 


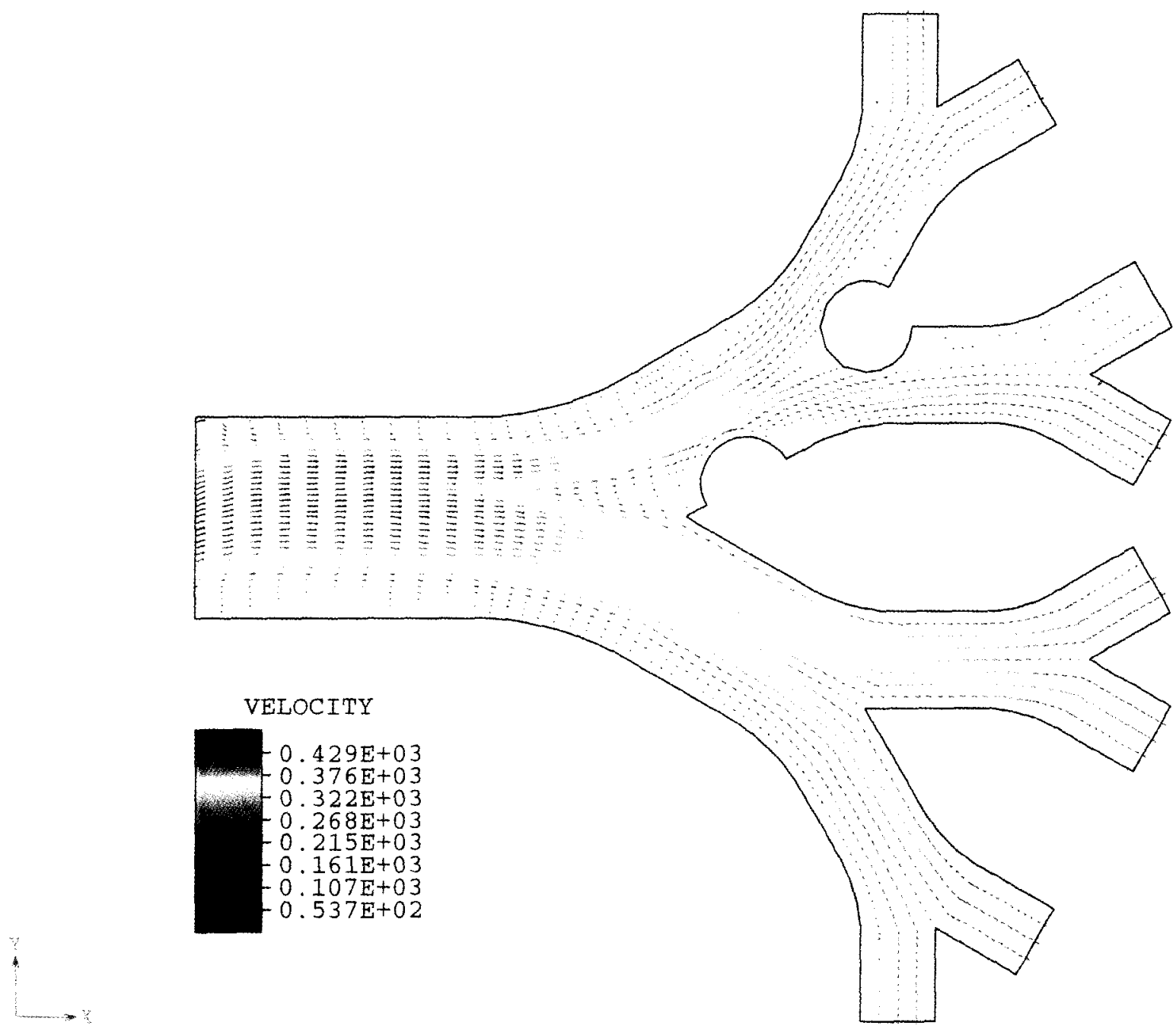

FIGURE 7 Velocity field in generations $2 \leq \mathbf{I} \leq 5$ of a four-year-old's lung with inlet velocity corresponding to a respiratory intensity level 3 breathing rate (see Table I). The tumor located on the carinal ridge has radius $0.131 \mathrm{~cm}$; the tumor located on the inside wall has a radius of $0.131 \mathrm{~cm}$ and is located $0.067 \mathrm{~cm}$ from the bifurcation point (See Color Plate VII at the back of this issue)

\section{Interactive Effects of Tumors}

As a final example, we consider a case with two tumors. Although we investigated a large number of tumor combinations only one case will be discussed, that being when one tumor was located on the carinal ridge and one tumor was located on the side wall (see Figure 13 in Part I and Figures 7-8 in Part II). This tumor combination will be used to show how changing initial mean velocities can affect flow patterns.

\section{Bulk effects}

In the L1 case (Figure 13, Part I), the flow pattern is similar to that observed in Figure 10, Part I, when only a carinal ridge tumor was present. In both figures, the flow in region $4^{d}$ had a maximum velocity equal to approximately $45 \%$ of the maximal velocity in region $4^{c}$. This was not true in the L3 or L5 cases. For example, in the L5 simulation (only a carinal ridge tumor) the maximum velocity in region $4^{d}$ was 


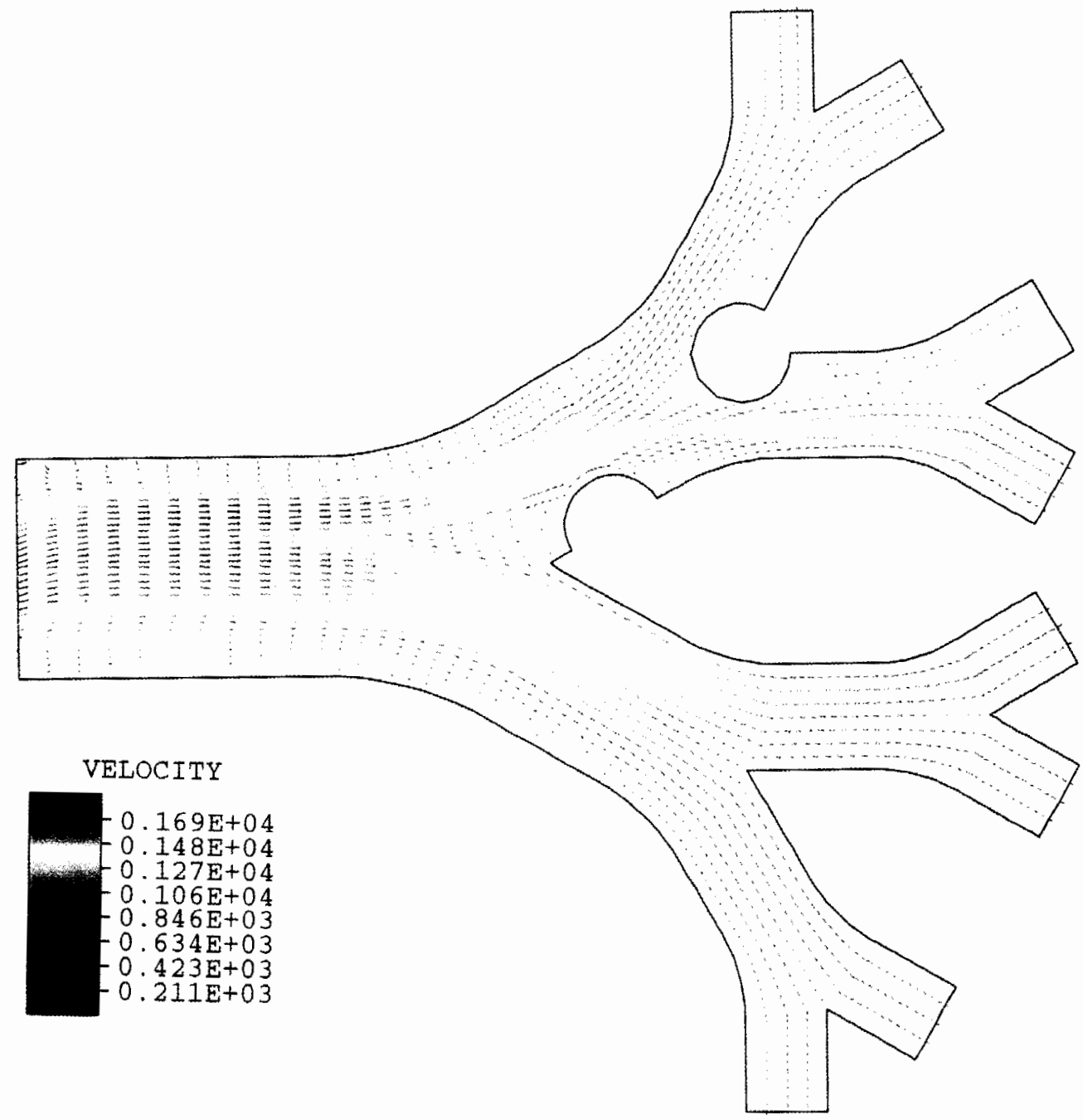

FIGURE 8 Velocity field in generations $2 \leq I \leq 5$ of a four-year-old's lung with inlet velocity corresponding to a respiratory intensity level 5 breathing rate (see Table I). The tumor located on the carinal ridge has radjus $0.131 \mathrm{~cm}$; the tumor located on the inside wall has a radius of $0.131 \mathrm{~cm}$ and is located $0.067 \mathrm{~cm}$ from the bifurcation point (See Color Plate VIII at the back of this issue)

$30 \%$ of that in region $4^{c}$ (see Figure 4 ); but, when there were two tumors present the maximal velocity in region $4^{d}$ was $75 \%$ of the maximal velocity in region $4^{c}$ (see Figure 8 ).

\section{Localized effects}

As the flow rate increased, an eddy (i.e., backflow) developed downstream from the carinal ridge tumor (Figure 9). The display is a close-up of the region directly behind the tumor. Backflow was also observed distal to the side wall tumor. Backflow was also experienced in the L 3 case. Decreasing the inlet flow rate decreased the size of the backflow region. Backflow was not observed in the L1 simulation.

\section{DISCUSSION}

The focus of this manuscript was to identify effects which could be related to differing respiratory intensities (as defined in Table I). 


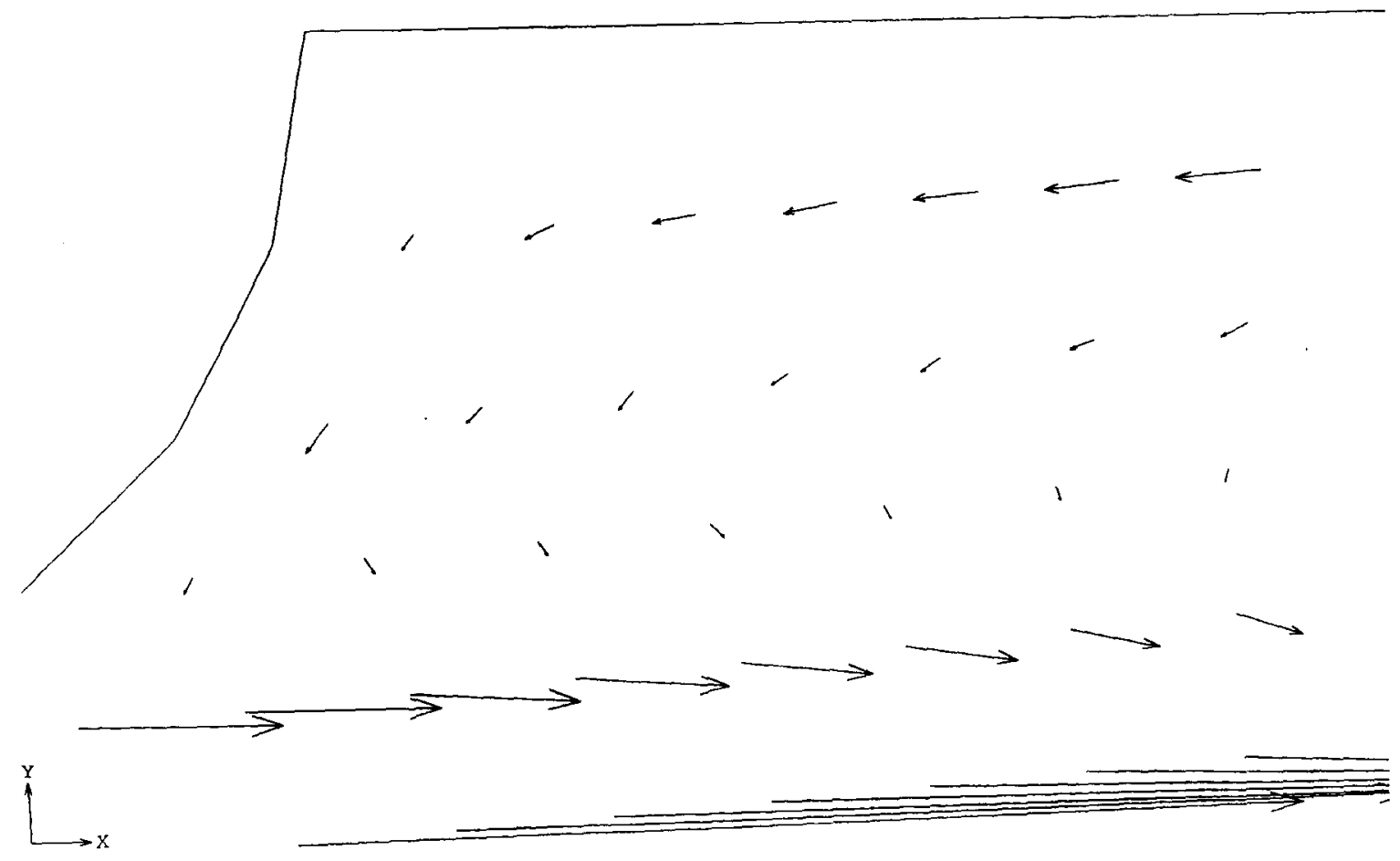

FIGURE 9 Velocity field in generation $I=3$ of a four-year-old's lung with inlet velocity corresponding to a respiratory intensity level 5 breathing rate (see Table I). The tumor located on the side wall has a radius of 0.131 and is located $0.067 \mathrm{~cm}$ from the bifurcation point. Notice the backflow present in this close up view of flow around the carinal ridge tumor

The condition when no tumors were present will be regarded as the control case. The major finding was that for the $\mathrm{L} 5$ case a maldistribution (i.e., redirection of flow) of flow occurred within the branching network (Figure 2), which could be attributed to the skewed character of the inlet velocity profile. The color-coded display shows that core flow is markedly shunted to airway $3^{a}$ at the expense of $3^{b}$. The maldistribution was not observed for the lower respiratory intensities; namely L1 (Figure 9, Part I) and L3 (Figure 1, Part II).

We considered two cases with isolated tumors: tumors either on a carinal ridge or on an airway wall. Each tumor when considered separately had the effect of redistributing incoming core flow relative their respective control cases (Table II). However, a tumor placed on a carinal ridge clearly had the dominant effect as illustrated by contrasting Figure 3 (carinal ridge tumor) with Figure 5 (sidewall tumor). This effect was manifest in the distribution of bulk flow to downstream airways.

Fluid dynamic interactions between tumors were quite complex. It was difficult to make generalizations regarding effects on flow patterns; instead, the results had to be examined on a case-by-case basis. However, it was possible to categorize our findings on the basis of bulk versus localized effects. For instance, for the lowest respiratory intensity (L1) the additional presence of a sidewall tumor had a minimal effect on the flow pattern (i.e, it was not too different from the case of a carinal ridge tumor alone). But, when the respiratory intensity was increased to L3 and L5 the side wall tumor did play a role.

A very interesting feature of our computations simulating tumor interactions was the presence of eddies. These backflow currents were observed downstream 
from both carinal ridge tumors and sidewall tumors for the L3 and L5 cases. Figure 9 is a close-up of the situation distal to a carinal ridge tumor.

The fluid dynamic findings presented above have implications to factors affecting the deposition of inhaled pharmacologic drugs. Martonen (1993) has demonstrated that particle deposition can be formulated by superimposing the separate mechanisms of inertial impaction, sedimentation and diffusion. These respective mechanisms are active in all airways during a breathing cycle, but have differing efficiencies in different airways depending on aerosol characteristics, ventilatory parameters and airway morphologies. The findings presented herein suggest that drug particles may be preferentially deposited on the upstream surfaces of tumors due to enhanced efficiency of the inertial impaction mechanism which occurs when particles have sufficient velocities for their trajectories to deviate from fluid streamlines. Conversely, drug particles may be preferentially deposited on the downstream surfaces of tumors due to enhanced efficiencies of the diffusion mechanism for small (i.e., submicron particles) and the sedimentation mechanism for larger particles. The mechanisms of diffusion and sedimentation are functions of the residence times that particles spend in airways. The eddies downstream of tumors would cause particles to be trapped and allow more time for deposition to occur by diffusion and sedimentation. In future endeavors we intend to integrate these observations into improved aerosol therapy protocols by actually calculating particle trajectories and performing sensitivity analyses to determine which factors most affect the targeted delivery of inhaled pharmaceuticals.

\section{References}

[1] Catley, C.K., Caez, H.J. and Mersheimer, W. (1951). Primary bronchogenic carcinoma of the lung in children: Review of the literature: Report of a case. American Journal of Diseases in Childhood, 82, 49-69.

[2] Clements, R. and Gravelle, I.H. (1986). Laryngeal Papillomatosis. Clinical Radiology, 37, 547-550.

[3] Cleveland, R.H. (1979). Symmetry of Bronchial Angles in Children. Pediatric Radiology, 133, 89-93.

[4] Cohen, S.R., Seltzer, S., Geller, K.A. and Thompson, J.W. (1980). Papilioma of the larynx and tracheobronchial tree in children. Annals of Otology, Rhinology, and Laryngology, $89,497-503$

[5] Condon, V.R., Phillips, E.W. (1962). Bronchial adenoma in children: a review of the literature and report of three cases. Americal Journal of Roentgenology, 88, 543-554.

[6] dePareder, C.G., Pierce, W.S., Grolf, D.B. and Waldhausen J.A. (1970). Bronchogenic tumors in children. Archives of Surgery, 100, 574-576.

[7] Dhand, R. and Tobin, M.J. (1997). Inhaled bronchodilator therapy in mechanically ventilated patients. Americal Journal of Respiratory and Critical Care Medicine, 156, 3-10.

[8] FIDAP. (1993). Fluid Dynamics International. Evanston, Illinois.

[9] Hartman, G.E. and Shochat, S.J. (1983). Primary pulmonary neoplasms of childhood: A review. The Annals of Thoracic Surgery, 36, 108-119.

[10] Hofmann, W., Martonen, T.B., Graham, R.C. (1989). Predicted deposition of nonhygroscopic aerosols in the human lung as a function of subject age. Journal of Aerosol Medicine, 2, 49-68.

[11] Kirchner, J.A. (1951). Papilloma of the larynx with extensive lung involvement, Laryngoscope, 61, 1022-1029.

[12] Kramer, S.S., Wehunt, W.D., Stocker, J.T. and Kashima, H. (1985). Pulmonary Manifestations of Juvenile Laryngotracheal Papillomatosis. A.JR, 144, 687-694.

[13] Martonen, T.B., Graham, R.C. and Hoffman, W. (1989). Human subject age and activity level: Factors addressed in a biomathematical deposition program for extrapolation modeling. Health Physics, 57, 49-59. A.

[14] Martonen, T.B. (1993). Mathematical Model for the selective deposition of inhaled pharmaceuticals. Journal of Pharmaceutical Sciences, 82, 1191-1199.

[15] Morrison, G.A.J., Kotecha, B, and Evans, J.N.G. (1993) Ribavirin treatment for juvenile respiratory papillomatosis The Journal of Laryngology and Otology, 107, 423-426.

[16] Nunez, V.A., Spjut, H.J, and Rosenber, H.S. (1965). Bronchial adenomas in children. Texas State Journal of Medicine, 61, 683-686.

[17] Oho, K. and Amemiya, R. (1980). Practical Fiberoptic Bronchoscopy. IGAKU-SHOIN Ltd., Tokyo, Japan.

[18] Oleszczuk-Raszke, K. and Cremin, B.J. (1988). Computed tomography in pulmonary papillomatosis. The British Journal of Radiology, 61, 160-161.

[19] Tatsumura, T., Koyama, S., Tsujimoto, M., Kitagawa, M. and Kagamimori, S. (1993). Further study of nebulisation chemotherapy, a new chemotherapeutic method in the treatment of lung carcinomas: fundamental and clinical. British Joumal of Cancer, 68, 1146-1149.

[20] Vawter G.F. and Ferguson, C.F. (1958). Bronchial adenoma in childhood. Annals of Otology, Rhinology and Laryngol ogy, 67, 1113-1125.

[21] Wang, L.T., Wilkins, E.W. and Bode, H.H. (1993). Bronchial carcinoid tumors in pediatric patients. Chest, 103, 14261428.

[22] Weibel, E.R. (1963). Morphometry of the human lung. Academic Press, New York.

[23] Weisel, W. and Leplye, D. (1961). Tracheal and bronchial adenomas in childhood. Pediatrics, 28, 394-398.

[24] Wellons, H.A. Jr., Eggleston, P., Golden, G.T. and Allen, M.S. (1976) Bronchial adenomas in childhood. Two case reports and review of literature. American Journal of Diseases of Children, 130, 301-304. 


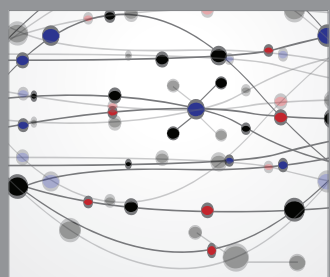

The Scientific World Journal
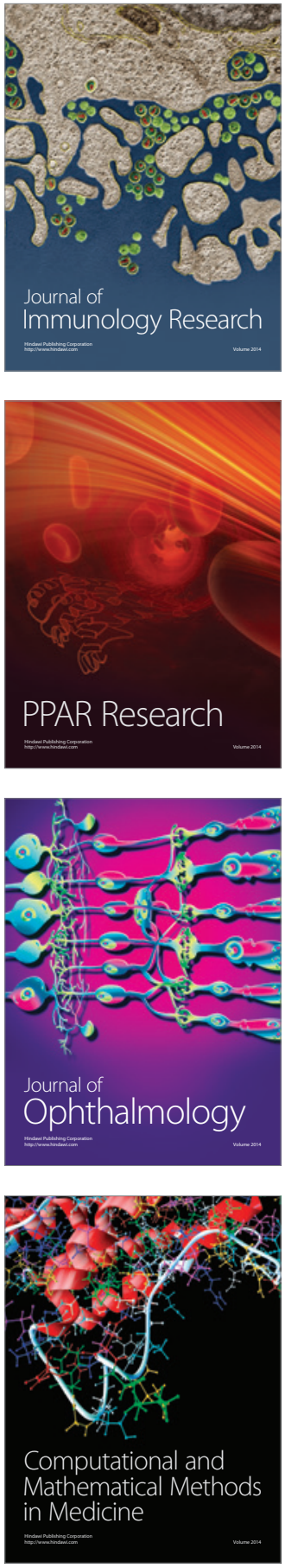

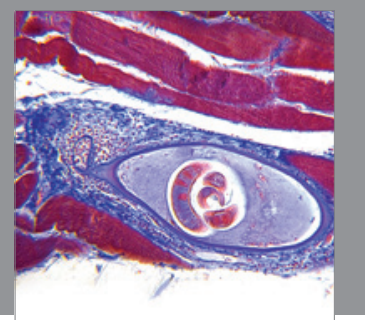

Gastroenterology

Research and Practice
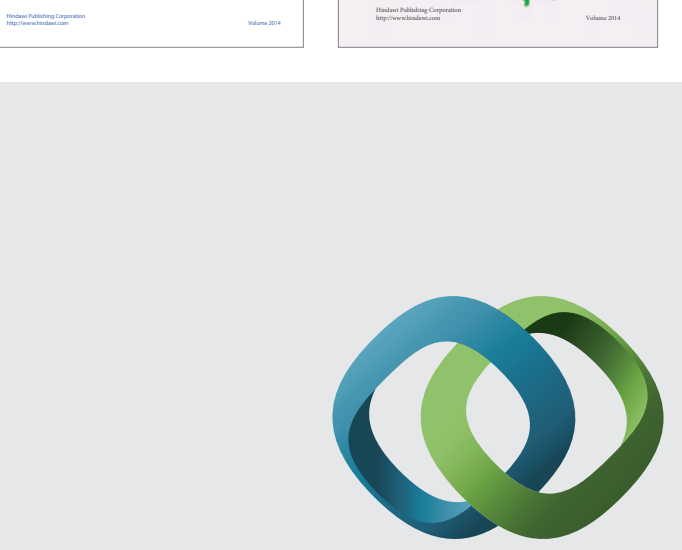

\section{Hindawi}

Submit your manuscripts at

http://www.hindawi.com
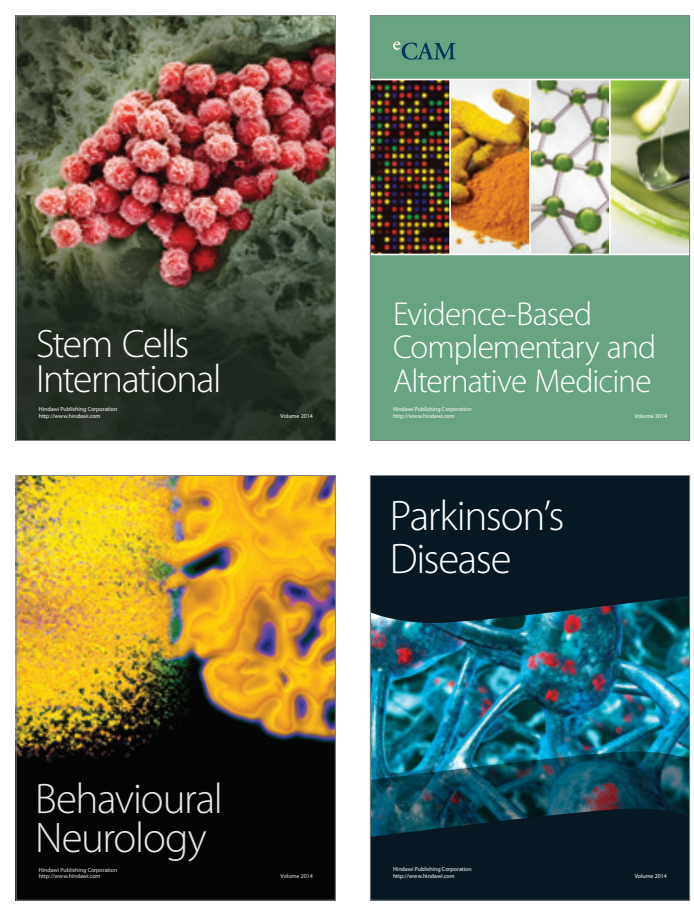

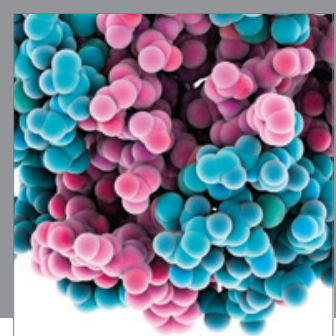

Journal of
Diabetes Research

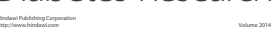

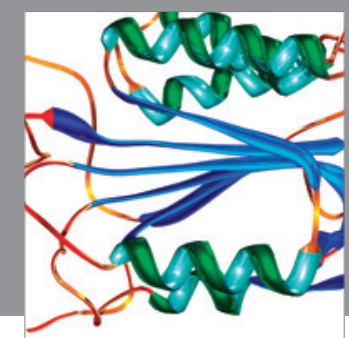

Disease Markers
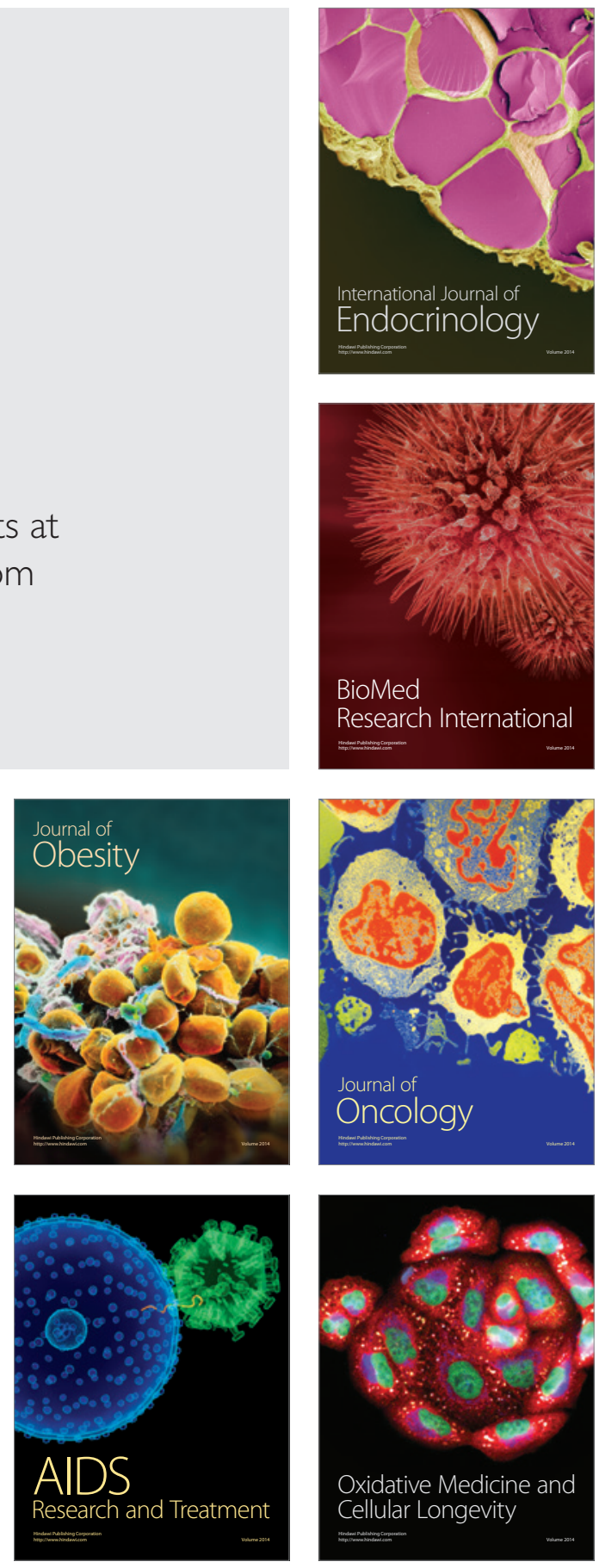